Article

\title{
Nearly Single-Cycle Terahertz Pulse Generation in Aperiodically Poled Lithium Niobate
}

\author{
Yuri Avetisyan $1, *\left(\mathbb{C}\right.$ and Masayoshi Tonouchi ${ }^{2}$ \\ 1 Microwave Engineering Department, Yerevan State University, Yerevan 0025, Armenia \\ 2 Institute of Laser Engineering, Osaka University, 2-6 Yamadaoka, Suita, Osaka 565-0871, Japan; \\ tonouchi@ile.osaka-u.ac.jp \\ * Correspondence: yuriav@ysu.am; Tel.: +374-10-56-8006
}

Received: 21 December 2018; Accepted: 24 January 2019; Published: 27 January 2019 updates

\begin{abstract}
In the present work, an opportunity of nearly single-cycle $\mathrm{THz}$ pulse generation in aperiodically poled lithium niobate (APPLN) crystal is studied. A radiating antenna model is used to simulate the THz generation from chirped APPLN crystal pumped by a sequence of femtosecond laser pulses with chirped delays $(m=1,2,3 \ldots)$ between adjacent pulses. It is shown that by appropriately choosing $\Delta t_{m}$, it is possible to obtain temporal overlap of all $\mathrm{THz}$ pulses generated from positive (or negative) domains. This results in the formation of a nearly single-cycle $\mathrm{THz}$ pulse if the chirp rate of domain length $\delta$ in the crystal is sufficiently large. In the opposite case, a few cycle $\mathrm{THz}$ pulses are generated with the number of the cycles depending on $\delta$. The closed-form expression for the $\mathrm{THz}$ pulse form is obtained. The peak $\mathrm{THz}$ electric field strength of $0.3 \mathrm{MV} / \mathrm{cm}$ is predicted for APPLN crystal pumped by a sequence of laser pulses with peak intensities of the separate pulse in the sequence of about $20 \mathrm{GW} / \mathrm{cm}^{2}$. By focusing the $\mathrm{THz}$ beam and increasing the pump power, the field strength can reach values in the order of few $\mathrm{MV} / \mathrm{cm}$.
\end{abstract}

Keywords: terahertz; ultrafast photonics; nonlinear optics; lithium niobate

\section{Introduction}

The number of applications that require intense terahertz $(\mathrm{THz})$ pulses has been rapidly increasing in recent years [1,2]. Optical rectification (OR) of the femtosecond laser pulses in lithium niobate (LN) crystals has emerged as the most powerful way to generate broadband, nearly-single-cycle THz pulses with spectra centered at frequencies below $1 \mathrm{THz}$. Single-cycle $\mathrm{THz}$ pulses with record energy of $0.4 \mathrm{~mJ}[3]$ and pump-to-THz conversion efficiency $\eta=3.8 \%$ [4] have been reported. To satisfy phase matching condition, the pump beam is reflected off a grating to acquire a tilted-pulse-front (TPF), which is then subsequently imaged onto the crystal using a lens or a telescope. However, imaging errors limit the effective length of the crystal [5,6]; therefore, further increase in $\mathrm{THz}$ generation performance is challenging. Attempts to mitigate this problem by using contacting grating [7], stair-step echelons [8,9], and a combination of stair-step echelon with reflecting grating [10] still do not result in $\mathrm{THz}$ generation with energies or efficiencies close to the abovementioned values. It is well established $[11,12]$ that the effective interaction length in semiconductor materials such as $\mathrm{ZnTe}, \mathrm{GaP}$, and GaAs can be significantly larger due to operation ability at smaller tilt angles $<30^{\circ}$ and lower THz-wave absorption at room temperature. However, to escape 2nd- and 3rd-order multiphoton pump absorption (MPA), these materials have to be pumped at longer wavelengths $\lambda_{p} \geq 1.7 \mu \mathrm{m}$, where it is still relatively challenging to obtain femtosecond laser pulses with the required high power.

It should be noted that MgO-doped LN crystals have the advantages of a large nonlinear coefficient (especially among the inorganic crystals), sufficiently wide bandgap (the lowest order effective MPA is four-photon absorption at the $1.03 \mu \mathrm{m}$ pump wavelength), high damage threshold and, finally, 
the ability for spatial sign-modulation of the nonlinear coefficient. The latter is important, as nowadays, high-power pulses can be used to pump periodically-poled lithium niobate (PPLN) crystals. The large area PPLN crystals with a cross section $3 \mathrm{~mm} \times 3 \mathrm{~mm}$ are commercially available, and even a crystal with dimensions $1 \mathrm{~cm} \times 1.5 \mathrm{~cm}$ was recently used for $\mathrm{THz}$ generation [13]. The ability to significantly reduce THz-waves absorption in PPLN crystal by cryogenic cooling makes it possible to use crystals with a length of about $2-3 \mathrm{~cm}[13,14]$. Therefore, investigations of alternative methods for nearly single-cycle broadband $\mathrm{THz}$ pulse generation in domain-engineered PPLN crystals are interesting.

It has been well established that the use of aperiodically-poled lithium niobate (APPLN) leads to the generation of broadband $\mathrm{THz}$ pulses [15-18]. However, its temporal form is not a nearly single-cycle $\mathrm{THz}$ pulse (i.e., it is not determined by the spectral bandwidth) because different spectral components are radiated from different positions of the crystal. Like in the optical region, the technique of broadband pulse compression using Bragg grating, chirped mirror, and pairs of gratings or prisms, can be used. However, strong THz beam divergence limits its applications.

The opportunity of nearly single-cycle THz pulse generation in APPLN crystal in conjunction with a THz chirped mirror has been analyzed in detail by Yahaghi and co-worker in Ref. [19]. However, the difficulties of $\mathrm{THz}$ chirped mirror fabrication and its application in $\mathrm{THz}$ generator schemes complicates practical implementation. To avoid this problem, a nearly single-cycle $\mathrm{THz}$ pulse generation in chirped APPLN crystal using a pair of chirped optical pump pulses with various chirp rates was recently proposed and analyzed [20]. The relative chirp between the pump pulses compensates the temporal shift between $\mathrm{THz}$ frequencies generated at different locations in the crystal. As a consequence, the THz pulse emerges compressed upon exiting the crystal.

Taking advantage of the above idea, here, we introduce a new scheme to generate $\mathrm{THz}$ pulses with controllable number of $\mathrm{THz}$ field oscillations, from nearly single- to multi-cycles and, correspondingly, on the spectral bandwidth from broadband to narrowband. In contrast to the laser pulse format used in [20], here, the sequence of transform-limited laser pulses with chirped delay between adjacent pulses (so-called pulse-position modulated (PPM) signal in optical communication) is considered as the pump wave. Such sequences of laser pulses can be obtained by using a birefringent crystal array [21] or modern pulse shaping techniques [22].

The operation principle is similar to that presented in [20]; it is physically based on the fact that the temporary overlap of a sufficiently large number of phase-locked spectral components leads to the formation of an ultrashort pulse. It is well-known [16-18] that in the case of APPLN crystal pumping by single transform-limited laser pulse the generated $\mathrm{THz}$ waveform corresponds to domain structure of the crystal. More exactly, it corresponds to spatial reversal replica (or so-called phase-conjugate replica) of the APPLN domain lengths distribution, considering that the exciting laser pulse propagates faster than the radiated $\mathrm{THz}$-wave. The set of these temporally-shifted replicas is originated when the pump wave is the sequence of laser pulses with delays $\Delta t_{m}(m=1,2,3 \ldots M)$. By appropriately choosing the $\Delta t_{m}$, it is possible to obtain a temporary overlap of all generated frequencies (in the range of $\Delta \omega_{T H z}$ ) that results in the formation of a nearly single-cycle THz pulse in the case of sufficiently large $M$ and $\Delta \omega_{T H z}$, where $\Delta \omega_{T H z}$ is the bandwidth of THz generation in APPLN by separate laser pulse.

This paper provides a simple theoretical model for the efficient generation of a nearly single-cycle $\mathrm{THz}$ pulse in the chirped APPLN crystal pumped by a sequence of laser pulses with chirped delay times. Closed-form expression for the spectrum and waveform of the generated $\mathrm{THz}$ pulse is obtained. A peak $\mathrm{THz}$ electric field strength of $0.3 \mathrm{MV} / \mathrm{cm}$ is predicted for the APPLN crystal pumped by the sequence of laser pulses with peak intensity of the separate pulse in the sequence about $20 \mathrm{GW} / \mathrm{cm}^{2}$. By focusing the $\mathrm{THz}$ beam and using higher pump power, the electric field strength can be increased by an order of magnitude.

\section{Theoretical Model}

Let us consider a transform-limited optical pulse with Gaussian temporal and spatial profiles that propagates along the $x$-axis of APPLN crystal having a nonuniform distribution of the nonlinear 
coefficient $d_{33}=d_{33}(x)$. The APPLN crystal consists of $N$ number domains having lengths of $l_{k}(k=$ $1,2 \ldots N)$, which are reduced along the direction of the pump beam propagation. In every adjacent domain the sign of the nonlinear coefficient $d_{33}$ is opposite (Figure 1).

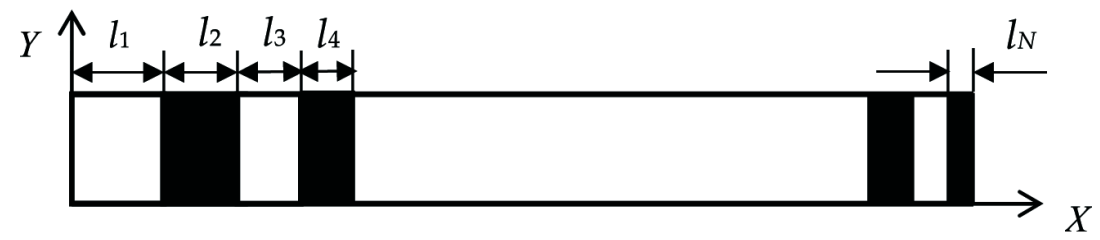

Figure 1. The schematic illustration of APPLN crystal, where white and dark colors are used for regions with opposite sign of nonlinear coefficient $d_{33}$.

To calculate the electric field of $\mathrm{THz}$ generation, the radiating antenna model $[23,24]$ is used. For simplicity, pump pulse depletion and its spatial and temporal distortions during propagation in the crystal are neglected. To avoid optical and $\mathrm{THz}$ wave reflections at the interfaces of the crystal, we assume that it is incorporated in a linear medium having the same refractive index. With these assumptions, the instantaneous intensity of the pump pulse is given by

$$
I(t, \vec{r})=I_{0} \exp \left[-\left(\frac{y^{2}+z^{2}}{a^{2}}\right)\right] \exp \left[-\left(\frac{t-x n_{g} / c}{\sigma}\right)^{2}\right]
$$

where $a$ is the beam waist radius, $n_{\mathrm{g}}$ is the group refractive index, $c$ is the velocity of light, $2 \sigma$ is the pulse duration at the $1 / e$ level that is related to the full width at half maximum by $\sigma_{\mathrm{FWHM}}=2(\ln 2)^{1 / 2} \sigma$, and $I_{0}$ is the peak on-axis intensity at entrance of the crystal $x=0$, which is related to pulse energy $\Im$ by $I_{0}=\Im / \pi^{3 / 2} a^{2} \sigma$.

It is known [25] that in the frequency domain low-frequency nonlinear polarization $P_{N L}$ is related to the pump intensity by the following expression:

$$
P_{N L}(\omega, \vec{r})=\frac{2 d_{33}(x)}{n_{0} c} f(y, z) I(\omega) e^{-i k_{g} x},
$$

where $f(y, z)=\exp \left[-\left(y^{2}+z^{2}\right) / a^{2}\right], I(\omega)=I_{0} \sqrt{\pi} \sigma \exp \left(-\omega^{2} \sigma^{2} / 4\right)$ is the Fourier transform of the on-axis intensity $I(t)=I_{0} \exp \left(-t^{2} / \sigma^{2}\right)$ at $x=0, n_{0}$ is the refractive index at the laser frequency, and $k_{g}$ $=\omega n_{g} / c$.

To calculate the THz far-field produced by the nonlinear polarization, the APPLN crystal is considered as an antenna fed by the $z$-oriented current given by $j_{z}(\omega, \vec{r})=i \omega P_{N L}(\omega, \vec{r})$. In antenna theory, the calculation of the radiation field for a given distribution of excitation currents leads to the integration in the so-called radiation integral over spatial coordinates $\vec{r} \equiv(x, y, z)$ [26]. To simplify the integration over transverse coordinates $y$ and $z$, it is assumed that the pump beam is completely inside the crystal. Finally, by presenting THz field as the superposition of fields radiated by the separate crystal domains, we determined that the $\mathrm{THz}$ field is given by

$$
E(\omega)=A_{0} i \omega I(\omega) e^{-i \omega t_{0}} \sum_{m=1}^{N}(-1)^{m+1}\left[e^{i \omega \tau_{m}}-e^{i \omega \tau_{m+1}}\right]
$$

where

$$
A_{0}=\frac{\mu_{0} d_{33} S}{2 \pi R n_{0} \Delta n}, t_{0}=\frac{n_{T H z}(R+L)}{c}, \tau_{m}=\frac{\Delta n}{c} \sum_{k=1}^{m-1} l_{k} \text { with } \tau_{1}=0,
$$

$\mu_{0}$ is the permeability of vacuum, $S=\pi a^{2}$ is the pump beam area, $l_{k}$ is the length of separate $k$-domain of the APPLN crystal, $n_{T H z}$ is the refractive index of the crystal at THz frequencies, $\Delta n=n_{T H z}-n_{g}$ is 
the refractive indexes mismatch, $L$ is the length of the crystal, and $R$ is the distance between the point of $\mathrm{THz}$ field observation and the exit surface of the APPLN crystal.

From the above, it follows that the temporal form of the radiated $\mathrm{THz}$ pulse can be easily calculated. Indeed, the factor $i \omega I(\omega)$ is the Fourier transform of temporal derivative of the intensity $\dot{I}(t)=(d I / d t)$, the exponential factors in the sum represent temporal shifts of $I(t)$ on the corresponding values, and the exponential factor before the summation symbol is related to the time of $\mathrm{THz}$-wave propagation from the entrance surface of the crystal to a point of $\mathrm{THz}$ field observation. Hence, the THz electric field in the time domain is given by

$$
E\left(t_{r}\right)=A_{0} \sum_{m=1}^{N}(-1)^{m+1}\left[I\left(t+\tau_{m}\right)-I\left(t+\tau_{m+1}\right)\right]
$$

where $t_{r}=t-t_{0}$ is the retarded time.

The above equation can be easily modified for the case, when APPLN crystal is pumped by a sequence of the laser pulses with delays $\Delta t_{m}=\tau_{2 m-1}-\tau_{1}$ and $m=1,2,3 \ldots N / 2$. Using the superposition principle, the generated $\mathrm{THz}$ field can be expressed by

$$
E_{\Sigma}\left(t_{r}\right)=\sum_{m=1}^{N / 2} E\left(t_{r}-\Delta t_{m}\right)
$$

Thus, the THz pulse form and spectrum can be easily calculated for a given distribution of the domain length $l_{k}$ and pump pulse duration $\sigma$.

\section{Results and Discussions}

Let's consider linearly-chirped APPLN, in which the domain lengths are decreasing from the front to the end of the crystal as $l_{k}=\left[l_{1}-(k-1) \delta\right]$, where $\delta=l_{k}-l_{k+1}$ is the length difference of neighboring domains and $k=1,2, \ldots 32$. Note that central frequency radiated from the $k$-domain is inversely proportional to its length $l_{k}$ and, therefore, higher $\mathrm{THz}$ frequencies are generated closer to the exit surface of the crystal. Because THz absorption at higher frequencies is larger, such a design of the crystal is favorable to mitigating $\mathrm{THz}$-wave damping.

Using Equation (5), the calculated THz waveform in the crystal with $l_{1}=90 \mu \mathrm{m}, \delta=1.6 \mu \mathrm{m}$, and $\sigma$ $=0.4 \mathrm{ps}$ is shown in Figure 2. Also, in the same figure, we present the $\mathrm{THz}$ waveforms obtained for pump pulses delayed at $\Delta t_{m}=\tau_{2 m-1}-\tau_{1}$ for $m=2$ and $m=3$, respectively. It is seen that there is a temporary overlap of the fields generated by the first positive domain $k=1$ and the third (the fifth) domain, if they are excited with delays $\Delta t_{2}\left(\Delta t_{3}\right)$, respectively.

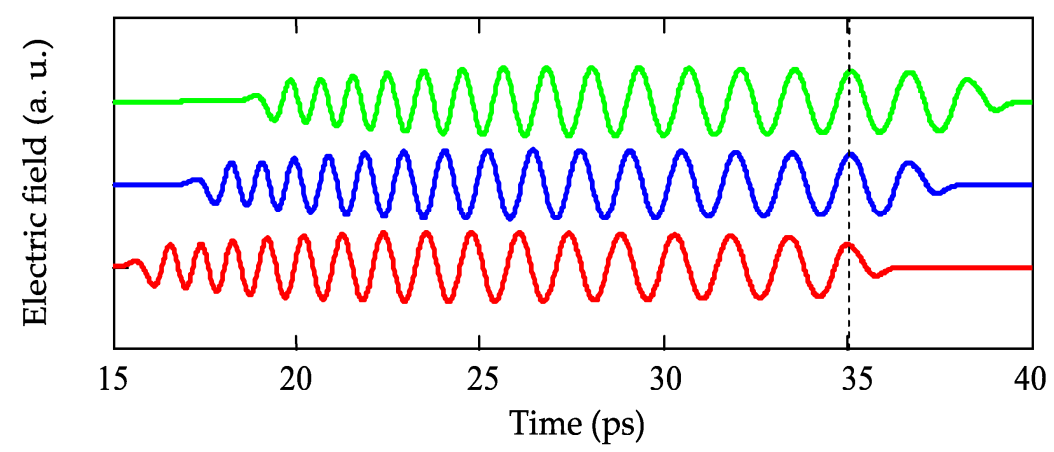

Figure 2. The temporal forms of THz pulses generated in the APPLN crystal by original laser pulse (red line) and its replicas delayed at $\Delta t_{2}$ (blue curve) and $\Delta t_{3}$ (green curve). The dashed line indicates nearly perfect overlapping of the fields radiated from the first three positive domains, when they are excited by laser pulses with the delays of $\Delta t_{1}=0, \Delta t_{2}$, and $\Delta t_{3}$. 
Therefore, temporary overlap of the fields generated by all positive domains will occur if the APPLN crystal is pumped by a sequence of laser pulses having delays $\Delta t_{m}$ with $m=1,2,3 \ldots N / 2$. As a result of the fields overlapping, a nearly single-cycle $\mathrm{THz}$ pulse can be formed, if the number of domains $N$ in the crystal and value of $\delta$ (characterizing the domain's length gradient) are sufficiently large. These conditions are necessary to obtain a broadband THz generation in APPLN crystal pumped by the single laser pulse. The use of a sequence of laser pulses with suitable delays $\Delta t_{m}$ is needed to satisfy the certain phase relationship for the spectral components of the broadband radiation. Obviously, in the case of relatively narrowband radiation (or small value of $\delta$ ), the temporary overlap of $\mathrm{THz}$ fields will lead to the formation of a few cycle $\mathrm{THz}$ pulses, with the number of the cycles depending on $\delta$.

The above statements are illustrated in Figure 3a, where using Equation (6), we present the temporal forms of THz pulse $E_{\Sigma}\left(t_{r}\right)$, excited by the sequence of pulses having delays $\Delta t_{m}(m=1,2,3$ ... 16) for cases of $\delta=1.6 \mu \mathrm{m}, \delta=0.8 \mu \mathrm{m}$, and $\delta=0.5 \mu \mathrm{m}$, respectively. The required sequence of laser pulses is schematically illustrated in Figure $3 \mathrm{~b}$ for cases of $\delta=1.6 \mu \mathrm{m}$ and $\delta=0.8 \mu \mathrm{m}$, respectively.

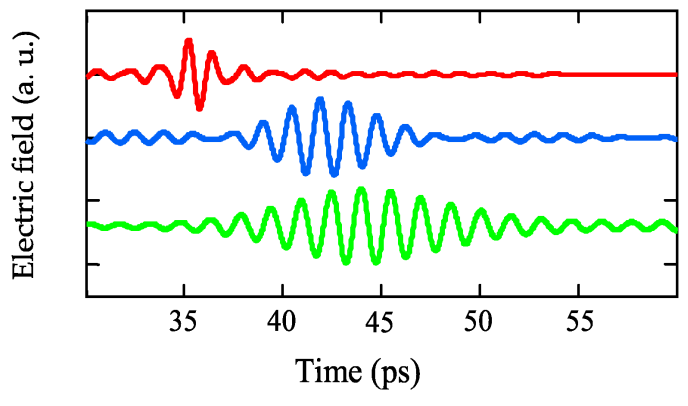

(a)

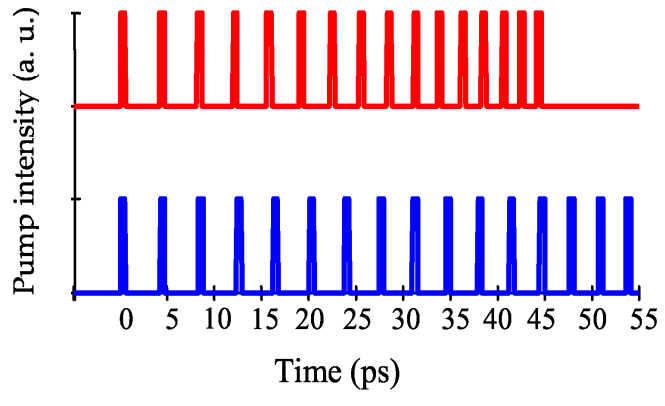

(b)

Figure 3. (a) Temporal forms of THz pulses generated in APPLN structures having different $\delta=1.6 \mu \mathrm{m}$ (red curve), $\delta=0.8 \mu \mathrm{m}$ (blue curve) and $\delta=0.5$ (green curve), respectively. The temporal forms are offset on the ordinate axis for clarity. In each case the crystal is pumped by the sequence of the laser pulses having delays $\Delta \tau_{m}$ with $m=1,2,3, \ldots$ 16. (b) Format of the pump pulses used for cases $\delta=1.6$ (red line) and $\delta=0.8$ (blue line), respectively.

For many applications, especially for THz-driven particle acceleration, the low-frequency part of the $\mathrm{THz}$ spectrum ( 0.3 to $1.5 \mathrm{THz}$ ) is most useful, because the longer wavelength compares well with typical sizes of particle bunches [10]. In our case, the THz spectrum is given by the product of the spectrum $E(\omega)$ generated by the first pump pulse and the factor $F=\left[1+\exp \left(-i \omega \Delta t_{2}\right)+\exp \left(-i \omega \Delta t_{3}\right)\right.$ $\left.+\ldots+\exp \left(-i \omega \Delta t_{M}\right)\right]$ representing application of the sequence of pump pulses with the delays $\Delta t_{m}$. The dependences of spectral amplitude $E_{\Sigma}(f)$ on frequency in the cases of $\delta=1.6 \mu \mathrm{m}$ and $\delta=0.8 \mu \mathrm{m}$ are presented in Figure 4. It is seen that the spectrum of the nearly single-cycle THz pulse $(\delta=1.6 \mu \mathrm{m})$ is broadband and centered mainly at frequencies lower than $1 \mathrm{THz}$. The narrowband multicycle $\mathrm{THz}$ radiation corresponds to the $\delta=0.8 \mu \mathrm{m}$.

It should be noted that all graphs presented in Figures $2-4$ are built with constant factor accuracy, i.e., arbitrary units are used for the values of functions. However, the absolute value of the field strength is very important parameter. By including the numerical value of $A_{0}$ in the calculations using Equations (5) and (6), we determine that the maximum electric field strength $E_{\Sigma \max }$ is estimated to be about $300 \mathrm{kV} / \mathrm{cm}$ at the distance $R=20 \mathrm{~mm}$, if the beam area $S$ and the peak intensity $I_{0}$ of every pulse in the sequence are $7 \mathrm{~mm}^{2}$ and $20 \mathrm{GW} / \mathrm{cm}^{2}$, respectively. Obviously, the estimated value can be increased by enhancing the pump power and by focusing the $\mathrm{THz}$ beam with a short focus lens. The choice of the numerical value for $I_{0}$ is related to the established fact that the generated $\mathrm{THz}$ field strength linearly depends on the pump intensity for up to $\sim 20 \mathrm{GW} / \mathrm{cm}^{2}$ [3]. As for the distance $R=$ $20 \mathrm{~mm}$, it satisfies the well-known condition of the far-field approximation [26]. 


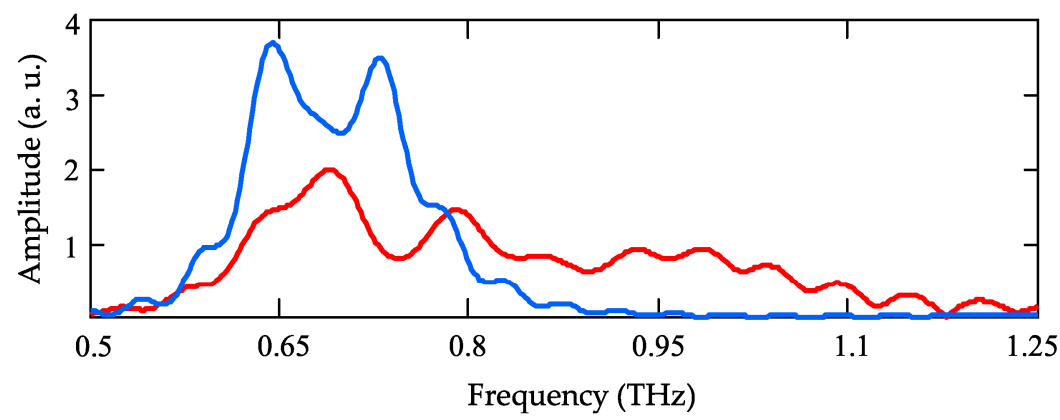

Figure 4. The spectra of THz generation in chirped APPLN crystal for the cases of $\delta=1.6 \mu \mathrm{m}$ (red curve) and $\delta=0.8 \mu \mathrm{m}$ (blue curve).

It is useful to note that there is a physically more transparent way to estimate peak electric field strength. It was already mentioned that $\mathrm{THz}$ pulses radiated by the first domain $E_{1}\left(t_{r}\right)$ and by all other positive domains are temporarily overlapped if the sequence of laser pulses with delays $\Delta t_{m}$ $=\tau_{2 m-1}-\tau_{1}(m=2,3 \ldots N / 2)$ is used. Therefore, the generated electric field can be approximately presented as $E_{\Sigma}\left(t_{r}\right) \approx N E_{1}\left(t_{r}\right) / 2$. Using expansion into a Taylor series, the first term of Equation (6) can be rewritten as

$$
E_{1}\left(t_{r}\right) \approx-A_{0} \tau_{2} \ddot{I}\left(t_{r}\right)
$$

where $\ddot{I}(t)$ is the second order derivative of the pump intensity.

Using above approximation, the maximum field strength can be given by $E_{\Sigma \max } \approx B_{0} N I_{0}$, where $B_{0}=\mu_{0} d_{33} S l_{1} / \pi \sigma^{2} R n_{0} c$. By substituting the numerical values used in the previous field estimation, we obtain $E_{\Sigma \max } \approx 320 \mathrm{kV} / \mathrm{cm}$, which is in good agreement with the earlier obtained result.

\section{Conclusions}

A simple theoretical model is developed for nearly single-cycle $\mathrm{THz}$ pulse generation in a chirped APPLN crystal pumped by a sequence of femtosecond laser pulses with appropriately chosen delays between adjacent pulses. The closed form expressions for $\mathrm{THz}$ pulse waveform and spectrum are obtained. The peak THz electric field strength of $0.3 \mathrm{MV} / \mathrm{cm}$ is predicted for APPLN crystal pumped by the sequence of laser pulses with peak intensities of the separate pulses in the sequence of about $20 \mathrm{GW} / \mathrm{cm}^{2}$. By focusing the $\mathrm{THz}$ beam and increasing the pump power, the field strength can reach values in the order of a few $\mathrm{MV} / \mathrm{cm}$. Such high-field $\mathrm{THz}$ radiation, having spectra centered at frequencies below $1 \mathrm{THz}$, could be an important tool for many applications.

Author Contributions: All authors equally contributed to the development of this work and the writing of the manuscript.

Funding: This research was partially funded by the JSPS KAKENHI Grant No. 17H01269, 18KK0140 and the Republic of Armenia State Committee of Science in the frames of the research project 18T-1C199.

Acknowledgments: The help of $\mathrm{H}$. Chosrowjan is acknowledged.

Conflicts of Interest: The authors declare no conflict of interest.

\section{References}

1. Hafez, H.A.; Chai, X.; Ibrahim, A.; Mondal, S.; Ferachou, D.; Ropagnol, X.; Ozaki, T. Intense terahertz radiation and their applications. J. Opt. 2016, 18, 093004. [CrossRef]

2. Zhang, X.C.; Shkurinov, A.; Zhang, Y. Extreme terahertz science. Nat. Photonics 2017, 11, 16-18. [CrossRef]

3. Fülöp, J.A.; Ollmann, Z.; Lombosi, C.; Skrobol, C.; Klingebiel, S.; Pálfalvi, L.; Krausz, F.; Karsch, S.; Hebling, J. Efficient generation of $\mathrm{THz}$ pulses with $0.4 \mathrm{~mJ}$ energy. Opt. Express 2014, 22, 20155-20163. [CrossRef] [PubMed] 
4. Huang, S.-W.; Granados, E.; Huang, W.R.; Hong, K.-H.; Zapata, L.E.; Kärtner, F.X. High conversion efficiency, high energy terahertz pulses by optical rectification in cryogenically cooled lithium niobate. Opt. Lett. 2013, 38, 796-798. [CrossRef] [PubMed]

5. Ravi, K.; Huang, W.R.; Carbajo, S.; Wu, X.; Kärtner, F.X. Limitations to THz generation by optical rectification using tilted pulse fronts. Opt. Express 2014, 22, 20239-20251. [CrossRef] [PubMed]

6. Zhang, B.; Li, S.; Chai, S.; Wu, X.; Ma, J.; Chen, L.; Li, Y. Nonlinear distortion and spatial dispersion of intense terahertz generation in lithium niobate via the tilted pulse front technique. Photonics Res. 2018, 6, 959-964. [CrossRef]

7. Yoshida, F.; Nagashima, K.; Tsubouchi, M.; Maruyama, M.; Ochi, Y. THz pulse generation using a contact grating device composed of $\mathrm{TiO}_{2} / \mathrm{SiO}_{2}$ thin films on LiNbO3 crystal. J. Appl. Phys. 2016, 120, 183103. [CrossRef]

8. Ofori-Okai, B.; Sivarajah, P.; Huang, W.; Nelson, K. THz generation using a reflective stair-step echelon. Opt. Express 2016, 24, 5057-5068. [CrossRef] [PubMed]

9. Avetisyan, Y.; Makaryan, A.; Tadevosyan, V.; Tonouchi, M. Design of a multistep phase mask for high-energy terahertz pulse generation by optical rectification. J. Infrared Millim. Terahz Waves 2017, 38, 1439-1447. [CrossRef]

10. Palfalvi, L.; Toth, G.; Tokodi, L.; Marton, Z.; Fulop, J.; Almasi, G.; Hebling, J. Numerical investigation of a scalable setup for efficient terahertz generation using a segmented tilted-pulse-front excitation. Opt. Express 2017, 25, 29560-29573. [CrossRef]

11. Polonyi, G.; Mechler, M.; Hebling, J.; Fulop, J. Prospects of semiconductor terahertz pulse sources. IEEE J. Sel. Top. Quantum Electron. 2017, 23, 8501208. [CrossRef]

12. Bakunov, M.I.; Bodrov, S.B.; Mashkovich, E.A. Terahertz generation with tilted-front laser pulses: Dynamic theory for low-absorbing crystals. J. Opt. Soc. Am. B 2011, 28, 1724-1734. [CrossRef]

13. Jolly, S.; Ahr, F.; Matlis, N.; Leroux, V.; Eichner, T.; Ravi, K.; Ishizuki, H.; Taira, T.; Kartner, F.; Maier, A. Towards millijoule narrowband terahertz generation using chirp-and-delay in periodically poled lithium niobite. In Proceedings of the High-Brightness Sources and Light-Driven Interactions, Strasbourg, France, 26-28 March 2018.

14. Ahr, F.; Jolly, S.; Matlis, N.; Carbajo, S.; Kroh, T.; Ravi, K.; Schimpf, D.; Schulte, J.; Ishizuki, H.; Taira, T.; et al. Narrowband terahertz generation with chirped-and-delayed laser pulses in periodically poled lithium niobate. Opt. Lett. 2017, 42, 2118-2121. [CrossRef] [PubMed]

15. Vodopyanov, K.L. Optical THz-wave generation with periodically-inverted GaAs. Laser Photon. Rev. 2008, 2, 11-25. [CrossRef]

16. Lee, Y.S.; Norris, T.B. Terahertz pulse shaping and optimal waveform generation in poled ferroelectric crystals. J. Opt. Soc. Am. B 2002, 19, 2791-2798. [CrossRef]

17. Kitaeva, G.K. Frequency conversion in aperiodic quasi-phase-matched structures. Phys. Rev. A 2007, 76, 043841. [CrossRef]

18. L'huillier, J.; Torosyan, G.; Theuer, M.; Rau, C.; Avetisyan, Y.; Beigang, R. Generation of THz radiation using bulk, periodically and aperiodically poled lithium niobate-Part 2: Experiment. Appl. Phys. B 2007, 86, 197-208. [CrossRef]

19. Yahaghi, A.; Ravi, K.; Fallahi, A.; Kärtner, F. Designing chirped aperiodically poled structures for high-energy single-cycle terahertz generation. J. Opt. Soc. Am. B 2017, 34, 590-600. [CrossRef]

20. Ravi, K.; Kartner, F. Generating compressed broadband terahertz pulses using aperiodically poled electro-optic crystals. arXiv 2018, arXiv:1710.07843v.2.

21. Dromey, B.; Zepf, M.; Landreman, M.; O’Keeffe, K.; Robinson, T.; Hooker, S.M. Generation of a train of ultrashort pulses from a compact birefringent crystal array. Appl. Opt. 2007, 46, 5142-5146. [CrossRef]

22. Salem, R.; Foster, M.A.; Gaeta, A.L. Application of space-time duality to ultrahigh-speed optical signal processing. Adv. Opt. Photonic 2013, 5, 274-317. [CrossRef]

23. L'huillier, J.; Torosyan, G.; Theuer, M.; Avetisyan, Y.; Beigang, R. Generation of THz radiation using bulk, periodically and aperiodically poled lithium niobate-Part 1: Theory. Appl. Phys. B 2007, 86, 185-196. [CrossRef]

24. Avetisyan, Y.; Zhang, C.; Tonouchi, M. Analysis of linewidth tunable terahertz wave generation in periodically poled lithium niobite. J. Infrared Millim. Terahz Waves 2012, 33, 989-998. [CrossRef] 
25. Schneider, A.; Neis, M.; Stillhart, M.; Ruiz, B.; Khan, R.; Günter, P. Generation of terahertz pulses through optical rectification in organic DAST crystals: Theory and experiment. J. Opt. Soc. Am. B 2006, 23, 1822-1835. [CrossRef]

26. Stutzman, W.L.; Thiele, G.A. Antenna Theory and Design; John Wiley \& Sons: Danvers, MA, USA, 2012.

(C) 2019 by the authors. Licensee MDPI, Basel, Switzerland. This article is an open access article distributed under the terms and conditions of the Creative Commons Attribution (CC BY) license (http:/ / creativecommons.org/licenses/by/4.0/). 